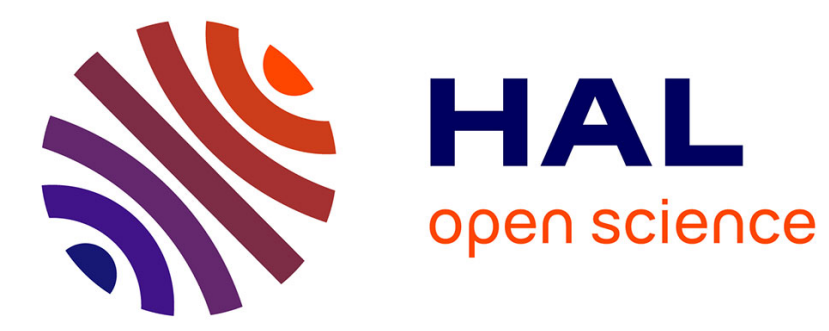

\title{
Lead Time Estimation Method for Complex Product Development Process
}

\author{
Hong-Bae Jun, Jin-Young Park, Hyo-Won Suh
}

\section{To cite this version:}

Hong-Bae Jun, Jin-Young Park, Hyo-Won Suh. Lead Time Estimation Method for Complex Product Development Process. Concurrent Engineering: Research and Applications, 2006, 14 (4), pp.313-328. 10.1177/1063293X06073302 . hal-00571199

\section{HAL Id: hal-00571199 \\ https://hal.science/hal-00571199}

Submitted on 1 Mar 2011

HAL is a multi-disciplinary open access archive for the deposit and dissemination of scientific research documents, whether they are published or not. The documents may come from teaching and research institutions in France or abroad, or from public or private research centers.
L'archive ouverte pluridisciplinaire HAL, est destinée au dépôt et à la diffusion de documents scientifiques de niveau recherche, publiés ou non, émanant des établissements d'enseignement et de recherche français ou étrangers, des laboratoires publics ou privés. 


\title{
Lead Time Estimation Method for Complex Product Development Process
}

\author{
Hong-Bae Jun, ${ }^{1, *}$ Jin-Young Park ${ }^{2}$ and Hyo-Won Suh ${ }^{2}$ \\ ${ }^{1}$ Laboratory of Computer-Aided Design and Production (STI-IPR-LICP) \\ Swiss Federal Institute of Technology in Lausanne (EPFL) \\ Station 9, ME B1, CH-1015 Lausanne, Switzerland \\ ${ }^{2}$ Department of Industrial Engineering, Korea Advanced Institute of Science \\ and Technology 373-1, Guseong-dong, Yuseong-gu Daejon \\ 305-701, Republic of Korea
}

\begin{abstract}
As the lifecycle of a product gets shorter, the timely introduction of new products becomes an invaluable asset to most manufacturing firms. It is needless to say that the competitive edge is given to a firm with the better knowledge on how to estimate the product development (PD) process because reducing the product development time is a critical issue for manufacturing firms. However, it is difficult to estimate the performance of the PD process because of its complexity. So far, in spite of its strategic importance, only a few studies have been done. To overcome this limitation, in this study, the authors provide a heuristic algorithm that can estimate the lead time of a complex PD process by series-parallel network transformation and network reduction methods with estimates of the makespans of branch-merge types. To show the usefulness of this method, computational experiments have been carried out.
\end{abstract}

Key Words: product development process, lead time, process pattern, series-parallel network.

\section{Introduction}

In general, a product development (PD) process is defined as a set of activities involved in taking a design problem during product development from setting initial specifications to producing a finished artifact that meets specifications [1,2]. It has a large number of decision-making activities that combine creative thinking, experience, intuition, and quantitative analysis, the characteristics of which are iterative, cooperative, evolutionary, and uncertain. These characteristics make the PD process complex. Figure 1 shows an example of a complex PD process of $K$ company that produces motor parts of an automotive.

As the PD process becomes more complex, managing a PD project in viewpoint of time becomes an important issue for managers. However, there exists a fundamental problem: how does one estimate the lead time of a PD process? This is a classic problem of the project management research area. Critical path method (CPM) [3] and program evaluation and review technique (PERT) $[3,4]$ are well-known approaches to solving this problem. Although they are well suited to analyze simple

*Author to whom correspondence should be addressed.

E-mail: hongbae.jun@epfl.ch
PD projects, they are not powerful enough for complex PD process because their assumptions are too strict to fully consider its complex structure [5].

Although some studies have been proposed to estimate the lead time of a complex PD process (or project), they still lack an effective model that handles various patterns of activities such as feedback, overlap, cycle, and so on. In this study, in order to overcome these limitations, the authors provide an analytically tractable algorithm that can estimate the lead time of a complex PD process. To estimate the lead time effectively, a complex PD process is formally defined as an activity network, and then this network is transformed into a series-parallel network. The lead time of this series-parallel network can be calculated by simplifying the network with formulae for estimating makespans of several activity patterns, which were defined in Jun et al. [6] (see Appendix).

The remainder of this study is organized as follows: related previous studies are described in the following section; the basic assumptions, notations, and definitions used in the heuristic algorithm presented in this study are introduced in Section 3; Section 4 describes the overview and detailed procedure of the heuristic algorithm; to give a better understanding of the authors' approach, an example is introduced in Section 5; and finally, in order to verify the effectiveness of the algorithm, the result of computational 
H.-B. JUN ET AL.

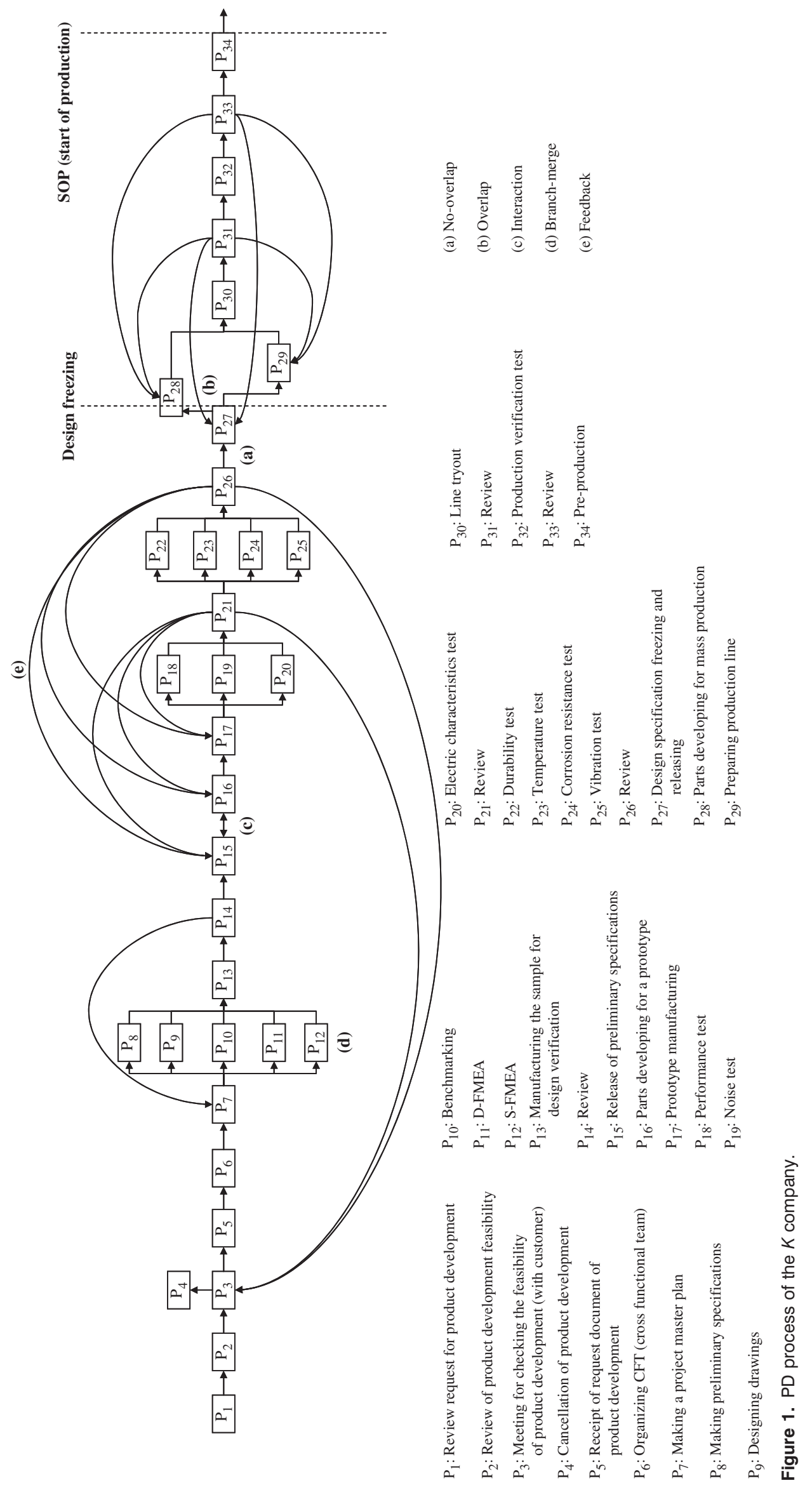


Table 1. Comparison of previous studies.

\begin{tabular}{|c|c|c|c|c|c|c|c|}
\hline \multirow{2}{*}{\multicolumn{2}{|c|}{ Classification }} & \multirow[b]{2}{*}{ Reference } & \multirow[b]{2}{*}{$\begin{array}{l}\text { Solution } \\
\text { approach }\end{array}$} & \multicolumn{4}{|c|}{ Relation } \\
\hline & & & & $R_{1}$ & $R_{2}$ & $\boldsymbol{R}_{3}$ & $\boldsymbol{R}_{\mathbf{4}}$ \\
\hline \multirow[t]{3}{*}{ Evaluation } & \multirow[t]{3}{*}{ Quantitative approach } & [18] & Continuous time Markov chain & - & - & - & - \\
\hline & & [21] & Signal flow graph & - & $\bullet$ & - & $\bullet$ \\
\hline & & {$[1,2]$} & Reward Markov chain & - & $\bullet$ & - & - \\
\hline \multirow[t]{10}{*}{ Evaluation and Optimization } & \multirow[t]{6}{*}{ Scheduling } & {$[17]$} & Heuristic algorithm & - & - & - & • \\
\hline & & {$[22]$} & Heuristic algorithm & - & - & $\bullet$ & - \\
\hline & & [15] & Reward Markov chain & - & $\bullet$ & - & $\bullet$ \\
\hline & & [22] & Heuristic algorithm & - & $\bullet$ & - & $\bullet$ \\
\hline & & [24] & Knowledge-based approach & - & - & - & - \\
\hline & & [25] & Heuristic algorithm & - & - & - & - \\
\hline & \multirow[t]{4}{*}{ Concurrent } & {$[26]$} & Dynamic program & $\bullet$ & - & - & - \\
\hline & & {$[27]$} & Nonlinear program & $\bullet$ & - & - & - \\
\hline & & [28] & Probabilistic model & $\bullet^{a}$ & $\bullet^{\mathrm{a}}$ & - & - \\
\hline & & {$[11,29]$} & Nonlinear program & $\bullet$ & - & - & - \\
\hline
\end{tabular}

$R_{1}$ Overlap.

$R_{2}$ Iteration.

$R_{3}$ Branch and merge pattern (AND-AND type).

$R_{4} \quad$ Branch and merge pattern that allows alternative routing

a (Independent, dependent, interdependent) $\times($ Sequential, partial overlapping, concurrent)

experiments on a real example and randomly generated examples is presented in Section 6.

\section{Previous Research}

There have been some methods for predicting a lead time of a project such as PERT and CPM [5]. CPM estimates a lead time by simply summing deterministic durations of activities in the critical path. On the contrary, PERT evaluates a lead time of a project under the assumption that each activity has a stochastic duration time [7,8]. Although $\mathrm{CPM}$ and PERT are well-known project management methods, they have fundamental limitations in considering complex PD projects for the following reasons:

1. PERT/CPM cannot handle complex patterns among activities, for example, iterative pattern, overlap pattern [9], multiple branching pattern [10], probabilistic branching pattern [10], and so on, because they are directed and acyclic graphs [11-14].

2. PERT/CPM assume that no activity can begin until all preceding activities have been completed. Because of this assumption, they allow only an AND-synchronous routing rule at branching and merging, which is not appropriate for describing many practical situations.

3. PERT requires an exact distribution function for the duration of each activity. However, it is not easy to obtain such a function [15].

To overcome the limitation of PERT, graphical evaluation and review technique (GERT) [16,17] has been proposed. Furthermore, some studies dealt with estimation methods of PD process as shown in Table 1. For example, Belhe and Kusiak [18] used a continuous time Markov chain method with a timed Petri net model in order to assess the total completion time of the whole PD process under resource constraints. Johnson et al. [2] also suggested a Markov-based model with revised design structure matrix (DSM) for measuring the approximation of the execution time of an iterative PD process. In addition, Smith and Eppinger [15] used a reward Markov chain method to compute the lead time in a sequential iterative PD process. On the other hand, Kara et al. [19] proposed an estimating method of PD project completion time with a heuristic scheduling algorithm considering resource constraints in a multiproject $\mathrm{CE}$ environment. They considered the unique characteristics of CE projects, such as concurrency and dual-level project management structure. Moreover, Yan et al. [20] modeled product-process design activity pairs as a network in order to deal with specific PD characteristics, such as design iterations. Based on it, they proposed a method for computing the mean duration of the activity pair.

In spite of many previous studies, they are limited in terms of estimating the lead time of complex PD process. Many techniques such as GERT, Markov-based models, and signal flow graph do not deal with the performance analysis of the complex PD project that has overlap and branch-merge patterns with synchronization time rule, as shown in Table 2 . Furthermore, they are not able to completely analyze the complex network that has alternative routings because they cannot fully represent the alternative routing rule. 
Table 2. Comparison of project evaluation methods.

\begin{tabular}{|c|c|c|c|c|c|c|}
\hline Comparison criteria & PERT ([22]) & GERT ([16]) & Signal flow graph ([21]) & Markov chain $([1,18])$ & WTM ([23]) & PDP-network \\
\hline Iterative works & - & $\bullet$ & $\bullet$ & $\bullet$ & $\bullet$ & $\bullet$ \\
\hline Overlap & - & - & - & - & - & $\bullet$ \\
\hline Multi-parallel branch-merge & $\bullet$ & $\bullet$ & $\bullet$ & $\bullet$ & - & - \\
\hline Alternative routing & - & $\bullet$ & $\bullet$ & $\bullet$ & $\bullet$ & $\bullet$ \\
\hline Probabilistic branching & - & - & - & - & - & - \\
\hline Synchronization time rule & - & - & - & - & - & - \\
\hline Time distribution of an activity & $\bullet$ & $\bullet$ & $\bullet$ & $\bullet$ & - & - \\
\hline
\end{tabular}

To overcome these limitations, the authors propose a heuristic algorithm for estimating the lead time of the complex PD process. The PD process considered here includes overlap, cycle, feedback, communication, and branch-merge patterns with synchronization time rule and alternative routing. For more detailed information about each pattern and its analytic formula to estimate the makespan, see Appendix and refer to Jun et al. [6]. In this study, the authors focus on how efficiently the lead time of the whole PD process that has the above patterns is estimated.

\section{Product Development Project Network}

In this study, the following notations are used.

$A \quad$ Set of activities $A=\left\{A_{1}, A_{2}, \ldots, A_{n}\right\}$

$D_{i} \quad$ Duration of an activity $A_{i}, A_{i} \in A, i=\{1,2, \ldots, n\}$

$O \quad$ Set of operators, $O=\left\{O_{x}, O_{I}, O_{\mathrm{S}}, O_{\mathrm{F}}, O_{C 1}, O_{C 2}\right\}$

$O_{x} \quad$ Operator for no-overlap and overlap pattern that have the degree of overlapping, $x,(0 \leq x \leq 1$, $x=0$ : no-overlap, $x=1$ : completely overlap)

$O_{I} \quad$ Operator for the interaction pattern

$O_{\mathrm{S}} \quad$ Operator for the branch-merge pattern $O_{\mathrm{S}}=\left\{{ }_{n} S_{k},{ }_{n} S_{k}^{\prime},{ }_{n} M_{k},{ }_{n} M_{k}^{\prime}, k=1,2, \ldots, n\right\}$ where ${ }_{n} S_{k}$ and ${ }_{n} S_{k}^{\prime}$ are a synchronous operator and an asynchronous operator at branching, respectively, and ${ }_{n} M_{k}$ and ${ }_{n} M_{k}^{\prime}$ are a synchronous operator and an asynchronous operator at merging, respectively. Each represents selecting $k$ among $n$ paths.

$O_{\mathrm{F}} \quad$ Operator for the feedback pattern

$O_{C 1}$ Operator for the cycle pattern

$O_{C 2}$ Operator for the communication pattern

$M \quad$ Extended numerical design structure matrix

$\omega \quad$ Lead time of the PD process

$\Pi \quad$ Set of all paths of the PDP-network

$V \quad$ Set of nodes

$\Lambda \quad$ Set of arcs, $\Lambda=\left\{a_{1}, a_{2}, \ldots,\right\}$

$a_{i} \quad$ Element of $\Lambda$, i.e., an $\operatorname{arc}, i=\{1,2, \ldots$,

In addition, the following are assumed:

1. An activity has no self-loop.

2. There is no feedback that originates from the branch-merge operators.
3. An activity cannot be interrupted until it is completed.

4. Parameters such as duration of an activity, an iteration probability, and so on, can be estimated from historical data.

To handle a complex PD process, it is formally defined with a bi-directed, connected, and cyclic graph, called product development project-network (PDP-network).

Definition 1 PDP-network

The PDP-network is a bipartite multigraph, $G=(V, \Lambda)$, which should satisfy the following:

1. $V$ represents a set of nodes which can be partitioned into two disjoint sets, an activity $(A)$ and an operator $\left(O=\left\{O_{x}, O_{I}, O_{\mathrm{S}}, O_{\mathrm{F}}, O_{C 1}, O_{C 2}\right\}\right)$, such that $V=A \cup O$ and $A \cap O=\phi$.

2. $\Lambda=\left\{a_{1}, a_{2}, \ldots, a_{k}\right\}$. The $a_{i}$ s can be classified into three disjoint sets: $\Lambda_{1}, \Lambda_{2}$, and $\Lambda_{3}$ where $\Lambda_{1}$ is a set of uni-directed arcs, $a_{p} \mathrm{~s}$, such that $a_{p}=(i, j)$ with $(i \in A$ and $j \in O), \Lambda_{2}$ is a set of uni-directed arcs, $a_{q} \mathrm{~s}$, such that $a_{q}=(i, j)$ with $(i \in O$ and $j \in A)$, and $\Lambda_{3}$ is a set of bi-directed arcs, $a_{r} \mathrm{~s}$, such that $a_{r}=(i, j)$ with $(i \in A$ and $j \in O)$ or $(i \in O$ and $j \in A)$.

3. An operator can have multiple incoming or outgoing arcs.

4. $G$ contains a single start node (called source) that has no predecessors, and a single terminal node (called sink) that has no successors.

5. In the case that an activity is simply connected with the other activity in precedence order, the graphic representation of its operator is omitted for convenience.

As defined already, in the PDP-network, operators are used between activities in order to identify the relations between activities more clearly. Operators with six types represent six PD process patterns: overlap, interaction, feedback, cycle, communication, branching, and merging. Especially, branching and merging operators can represent several cases depending on combinations of routing and synchronization. For example, 


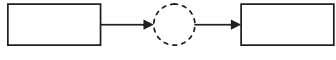

(a) Single (one-to one)

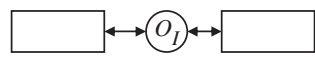

(c) Interdependent

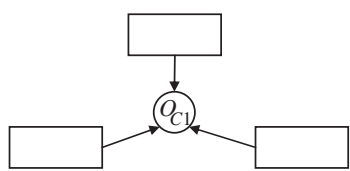

(e) Cycle

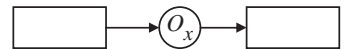

(b) Overlap

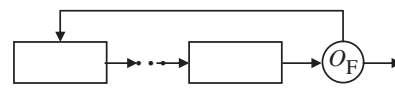

(d) Feedback

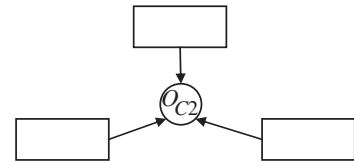

(f) Communication

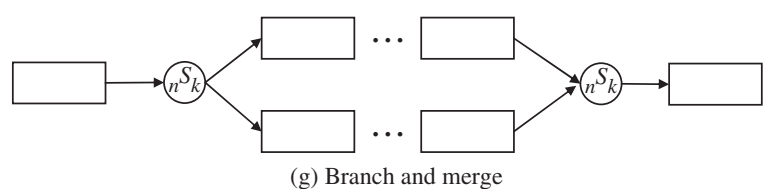

Figure 2. Type of patterns.

in the branch case, an operator is connected to multiple parallel activities if the branch node has AND routing rule $\left(O_{\mathrm{S}}={ }_{n} S_{n}\right)$. Meanwhile, a branching operator can select only one among several alternatives by XOR rule $\left(O_{\mathrm{S}}={ }_{n} S_{1}\right)$, or select more than two alternatives by selective choice (SOR) rule $\left(O_{\mathrm{S}}={ }_{n} S_{k}, k \neq 1, n\right)$. Each routing rule of the branch case has two synchronization types: synchronous and asynchronous, except for XOR routing rule which has only the asynchronous type. In the synchronous case, if all of the selected successors are ready, they start simultaneously. However, in the asynchronous case, if there is at least one ready successor among selected ones, they start. On the contrary, in the merge case, multiple parallel activities or multiple alternative activities can converge into one activity, according to their routing rule and synchronization type. The routing rules of the merge case are the same as those of the branch case. They also have two synchronization types. In the synchronous case, if all of the selected predecessors are completed simultaneously, then start the next activity. However, in the asynchronous case, if there is a completed predecessor among selected ones, then the next activity starts. One can describe many branch-merge patterns by combining branch patterns and merge patterns. However, not all combinations represent the genuine process logic in branch-merge patterns. For example, XOR-XOR and XOR-SOR patterns have no meaning. Ruling out these cases, one can classify branch-merge patterns into 21 types [6]. Figures 2 and 3 depict the patterns of PD process and an example of PDP-network, respectively.

To estimate the lead time of complex PDP-network efficiently, the PDP-network is converted into an extended numerical design structure matrix (ENDSM) defined as follows.
Definition 2 Extended Numerical Design Structure Matrix (ENDSM)

The ENDSM is defined as follows:

$$
M=\left(\begin{array}{cccc}
m_{00} & & \ldots & m_{0 n} \\
& m_{11} & \ldots & m_{1 n} \\
\vdots & \vdots & \ddots & \vdots \\
m_{n 0} & m_{n 1} & \ldots & m_{n n}
\end{array}\right), M \in R^{(n+1) \times(n+1)}
$$

$M$ should satisfy the following:

1. For any $i$ such that $1 \leq i \leq n$, row $i$ or column $i$ indicates an activity $i$.

2. $m_{i j}$ indicates

(a) type of branching or merging operator if $i=j$ $(1 \leq i=j \leq n)$. In this case, $m_{i j} \in O_{\mathrm{S}}={ }_{n} S_{k},{ }_{n} S_{k}^{\prime}$, $\left.{ }_{n} M_{k},{ }_{n} M_{k}^{\prime}, k=1, \ldots, n\right\}$.

(b) learning rate if $i=0$, for any $j$ such that $1 \leq j \leq n$. It is assumed that iterative process has the learning rate in doing a task repetitively.

(c) duration if $j=0$, for any $i$ such that $1 \leq i \leq n$.

(d) transition probability from an activity $i$ to an activity $j, \rho_{i j}$, such that $0 \leq \rho_{i j} \leq 1$, or the pattern type, $O \in\left\{O_{x}, O_{I}, O_{C 1}, O_{C 2}, 0<x \leq 1\right\}$, for all $i, j$ such that $1 \leq i<j \leq n$.

(e) iteration probability from an activity $i$ to an activity $j, \sigma_{i j}\left(0 \leq \sigma_{i j}<1\right)$, for all $i, j$ such that $1 \leq j<i \leq n$.

\section{Heuristic Algorithm}

Let $\Pi=\left\{\pi_{1}, \pi_{2}, \ldots, \pi_{t}\right\}$ denote the set of all paths of PDP-network from a source to a sink, where $t$ denotes the cardinality of $\Pi$, the number of paths. Then, the lead time of the PDP-network is simply expressed with $\omega=\max _{\pi_{i} \in \Pi}\left(\sum_{j \in \pi_{i}} D_{j}\right)$. However, it is not easy to estimate the lead time of the PDPnetwork because it has complex and numerous paths, which cause difficulty in determining a critical path. The complexity of PDP-network arises from the following reasons: First, the PDP-network is a cyclic and bi-directed graph. Second, it is composed of branching and merging patterns that have various synchronization and routing types. Finally, in the PDP-network, a downstream activity can start in advance with preliminary outputs of an upstream activity before the upstream activity is completed.

To tackle these difficulties, in this study, a heuristic algorithm is proposed that can estimate the lead time of a PDP-network. The overall procedure of this algorithm is as follows: At first, the whole 


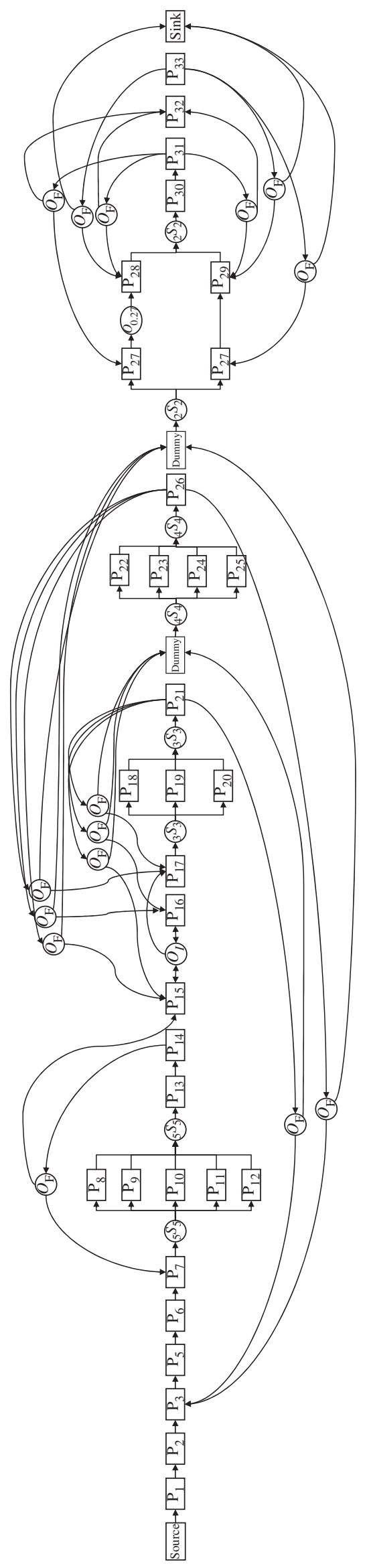

PD process is represented with the PDP-network. The PDP-network is simplified into a directed and acyclic network by reducing complex patterns such as overlap, cycle, and communication into simple dummy activities of which durations are estimated lead times of the complex patterns. With Markov chain analysis of the PDP-network, feedback patterns are also removed in the network. Although the simplified network has only branch-merge patterns, it is limited in terms of estimating the lead time of the whole PD process because of complex routing rules and synchronization types. However, if this network can be transformed into a series-parallel network [30] which consists of combinations of series and parallel activities, it is relatively easy to estimate the lead time. To this end, the simplified network is transformed into a series-parallel network using the following procedure: First, it is necessary to identify a branch-merge arc in the directed and acyclic network because this arc gives rise to Wheatstone's bridge [30] which is a cause of non-series-parallel network. The branch-merge arc can be defined as follows.

Definition 3 Branch-Merge Arc

The branch-merge arc is an arc $a_{k}=\left(i^{\prime}, j^{\prime}\right)$ such that $a_{k} \in \Lambda, \quad i^{\prime}, j^{\prime} \in A, \quad \sum_{i=1}^{n}\left\lceil m_{i j}\right\rceil \geq 2$, and $\sum_{j=1}^{n}\left\lceil m_{i j}\right\rceil \geq 2$. Here, $\left\lceil m_{i j}\right\rceil$ indicates the smallest integer number larger to $m_{i j}$.

Removing Wheatstone's bridge can be done by inserting appropriate dummy activities. This is called Wheatstone's bridge removing procedure in the proposed algorithm. After Wheatstone's bridge removing procedure, if there exist branch nodes not connected with related merge nodes as shown in Figure 4, then create dummy branch nodes in order to make a series-parallel network. This is called parallel-making procedure. After parallel-making procedure, the whole PDP-network is simplified into the network which consists of simple branch-merge patterns. The simple branch-merge pattern indicates the activity network which has parallel activities of only one phase between a branch activity and a merge activity (see Figure 4). Finally the overall lead time of the PDP-network is estimated by merging simple branch-merge patterns into single activity with their analytical formulae proposed in Jun et al. [6] until the series-parallel network is reduced into one activity.

The following is a heuristic algorithm (SBM-PDP) that specifies the above concept in detail. For convenience of handling, the PDP-network with ENDSM is described in the proposed algorithm.

\section{Algorithm: SBM-PDP}

Step 0. Describe the PDP-network of complex PD process and make its ENDSM. 


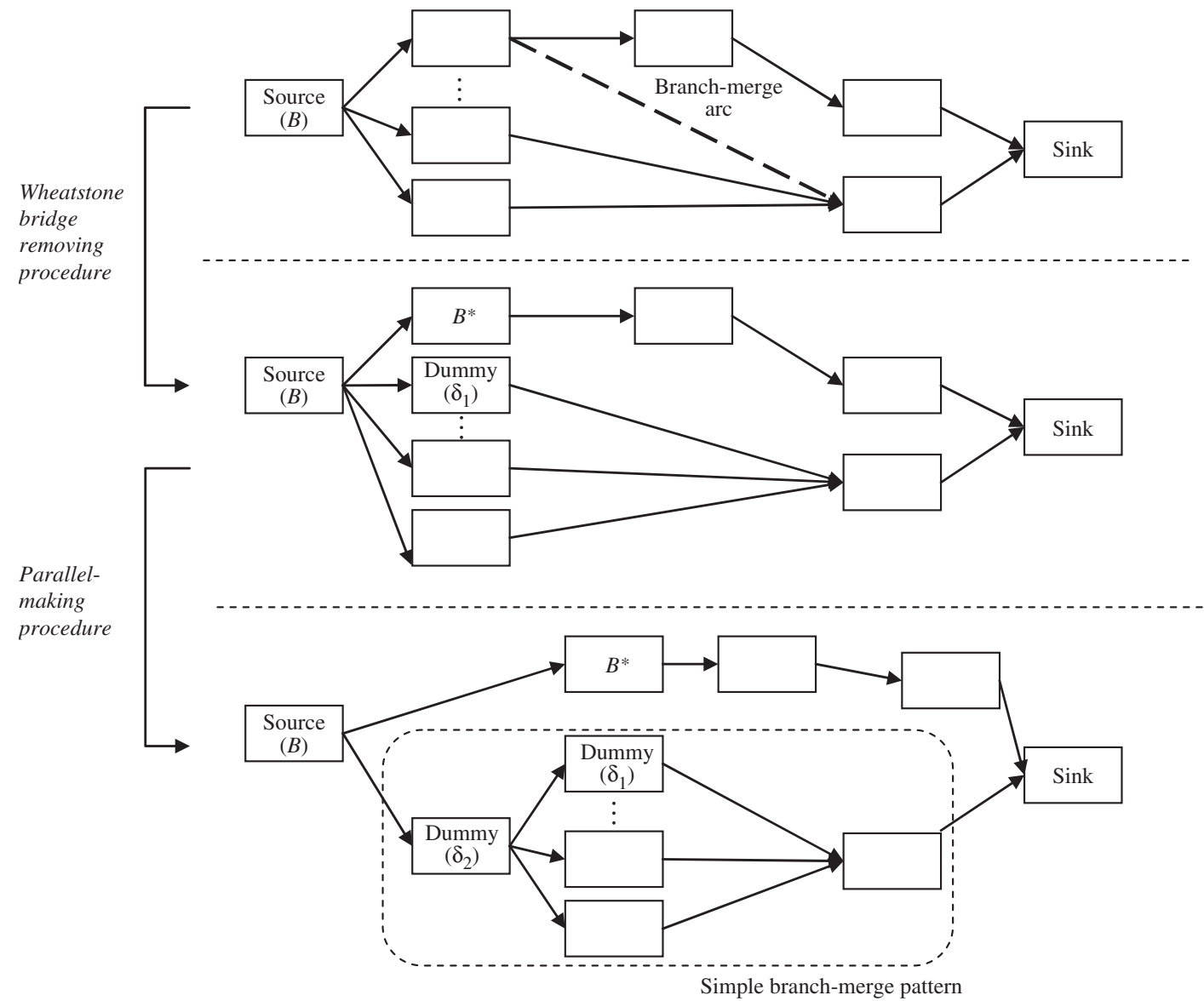

Figure 4. Series-parallel transformation and simple branch-merge pattern.

Step 1. Eliminate specific patterns, such as overlap, interaction, cycle, and communication, from the PDP-network.

For $i=1$ to $n$, do:

For $j=1$ to $n$, do:

(a) If $m_{i j}=O_{x}$ or $O_{I}$, then an activity $i$ has the overlap pattern or the interaction pattern with an activity $j$, respectively. In this case, use the following procedure to simplify the network.

1. Create a dummy node, $\delta$.

2. Calculate the duration $\left(D_{\text {new }}\right)$ of the pattern, using the analytic formula (refer to A.2 and A.3 of Appendix).

3 . Let $m_{\delta 0}=D_{\text {new }}$.

If $i$ is a branch node (or $j$ is a merge node),

4. Add the duration of the activity $i$ (or $j$ ) to the durations of branching activities except the activity $j$ (or $i$ ).

5. Hold the connectivity of the activity $j$ (or $i$ ). For this purpose, let $D_{i}=0, m_{i \delta}=1$, and for $k=1$ to $n, m_{\delta k}=m_{j k}$ (or $D_{j}=0, m_{\delta j}=1$, and for $k=1$ to $\left.n, m_{k \delta}=m_{k i}\right)$.

6. Eliminate $j$ (or $i$ ) from the matrix.
Otherwise,

4. Hold the connectivity of activity $i$ and $j$. For this purpose, let $m_{k \delta}=m_{k i}$ and $m_{\delta k}=m_{j k}$ for $k=1$ to $n$.

5. Eliminate $i$ and $j$ from the matrix.

(b) If $m_{i j}=O_{C 1}$ or $O_{C 2}$, then an activity $i$ and an activity $j$ are in the cycle pattern $\left(O_{C 1}\right)$ or in the communication pattern $\left(O_{C 2}\right)$. In this case, use the following procedure to simplify the network.

1. Create a dummy node, $\delta$.

2. Calculate the duration $\left(D_{\text {new }}\right)$ of the pattern, using the analytic formula (refer to A.4 and A.5 of Appendix).

3. Let $m_{\delta 0}=D_{\text {new }}$.

4. For $k=1$ to $n$, let $m_{k \delta}=m_{k p}$ and $m_{\delta k}=m_{p k}$ where $p$ indicates all is and $j$ s such that $m_{i j}=O_{C 1}$ or $O_{C 2}$.

5. Eliminate $p$ s from the matrix.

Step 2. If there exists the feedback pattern $\left(O_{\mathrm{F}}\right)$, i.e., there exists $\sigma_{i j}$ such that $\sigma_{i j}>0$ in the matrix $M$, then, eliminate it with the Markov chain analysis. 
1. Let $M^{\prime}$ be the diagonal matrix of $n$ size such that $m_{i i}^{\prime}$ means the duration of an activity $i$.

2. For each branch pattern with AND and SOR type, repeat the following procedure until all nested feedback patterns are considered.

1. Generate the transition probability matrix $P$. For this purpose, let the recurrent transition probability of a sink node be 1 .

2. Divide the matrix $P$ into canonical forms and calculate the average number of feedbacks from the following fundamental matrix.

$$
P_{A}=(1-Q)^{-1} \text {. }
$$

where $I$ is an identity matrix and $Q$ is a substochastic matrix. For more details, see Section 4.4 of Kao [31].

3. Calculate the duration of each activity.

$$
D=P_{A} \cdot M^{\prime} .
$$

For branch-merge patterns of the AND type, add up the above calculated value to each duration of the activity. In the case of the SOR type, add up the probabilistic value to each duration.

3. Replace $m_{i 0}$ with $D_{0 i}$ and eliminate the loops from the PDP-network.

1. For all $i$ such that $1 \leq i \leq n$, let $m_{i 0}=D_{0 i}$.

2. For all $i, j$ such that $1 \leq j<i \leq n$, let $m_{i j}=0$.

Step 3. Transform the PDP-network into the seriesparallel network with two transformation procedures.

(Wheatstone's bridge removing procedure)

For $i=1$ to $n$, and/or

For $j=1$ to $n$, if $a_{k}=\left(i^{*}, j^{*}\right)$ is the branch-merge arc, then,

1. If $m_{i^{*} q}=1, q \neq i^{*}$, and there exists a directed path from an activity $j^{*}$ to an activity $q$, then, let $m_{i^{*} q}=0$.

2. Otherwise, use the following procedure.

1. Let $m_{i^{*} j^{*}}=0$.

2. Create a dummy activity, $\delta_{1}$.

3. For the activity $\delta_{1}$, hold the pre-connectivity of the activity $i^{*}$. To get this, for $l=1$ to $n$, let $m_{l \delta_{1}}=m_{l l^{*}}$.

4. Let $m_{\delta_{1 j^{*}}}=1$ and eliminate $i^{*}$ from the matrix. (Parallel-making procedure)

For the branch node $B$ that has the branching activity, $B^{*}$,

1. Create a dummy activity, $\delta_{2}$.

2. For the activity $\delta_{2}$, hold the post-connectivity of the activity $B$. To get this, for $l=1$ to $n$, if $l \neq B^{*}$, then let $m_{\delta_{2} l}=m_{B l}$ and $m_{B l}=0$.

3. Let $m_{B \delta_{2}}=1$.
Step 4. Estimate the lead time of series-parallel network with analytic formulae of simple branchmerge patterns.

1. Use the following procedure until there does not exist any branch-merge pattern.

1. Detect branch-merge pattern.

2. Make the branch-merge pattern into the simple branch-merge pattern. To attain this, for the activity that is not directly connected with the branch or the merge activity, merge itself and other activities into one until the simple branch-merge pattern is made.

3. Calculate the duration $\left(D_{\delta}\right)$ of each simple branch-merge pattern with the predefined formula ([6]).

4. Reduce the simple branch-merge pattern into one activity. For this, create a dummy activity, $\delta$, and let the duration of the dummy activity $(\delta)$ be the duration of the simple branch-merge pattern, and then, hold the pre-connectivity of the branch node and the post-connectivity of the merge node. Finally, eliminate related activities of the simple branch-merge pattern.

2. Add up durations of the remaining activities. Then, the total summation is the estimated lead time of the PDP-network.

\section{Example}

In this section, in order to describe how to apply SBM-PDP to a real problem efficiently, a reduce-sized PD process of $K$ company is taken (Figure 5(a)). The $K$ company is a small-size manufacturing firm that produces automobile motors. Table 3 describes the necessary data of this example. The algorithm is applied to this example as follows. Figure 5 depicts the overall procedure.

Step 0. Describe the PDP-network of the example and make its ENDSM. At first, the PD process with the PDP-network is represented and described with ENDSM.

Step 1. Eliminate interaction, overlap, and cycle. To do this, the makespans of the interaction pattern have been estimated including Activities 5 and 6, overlap pattern including Activities 11 and 12, and cycle pattern including the Activities 13-15 with the proposed formulae in Jun et al. [6]. Subsequently, each pattern is replaced into single activity having the duration of estimated makespan. Figure 5(a) describes the simplified PDP-network before and after Step 2. 


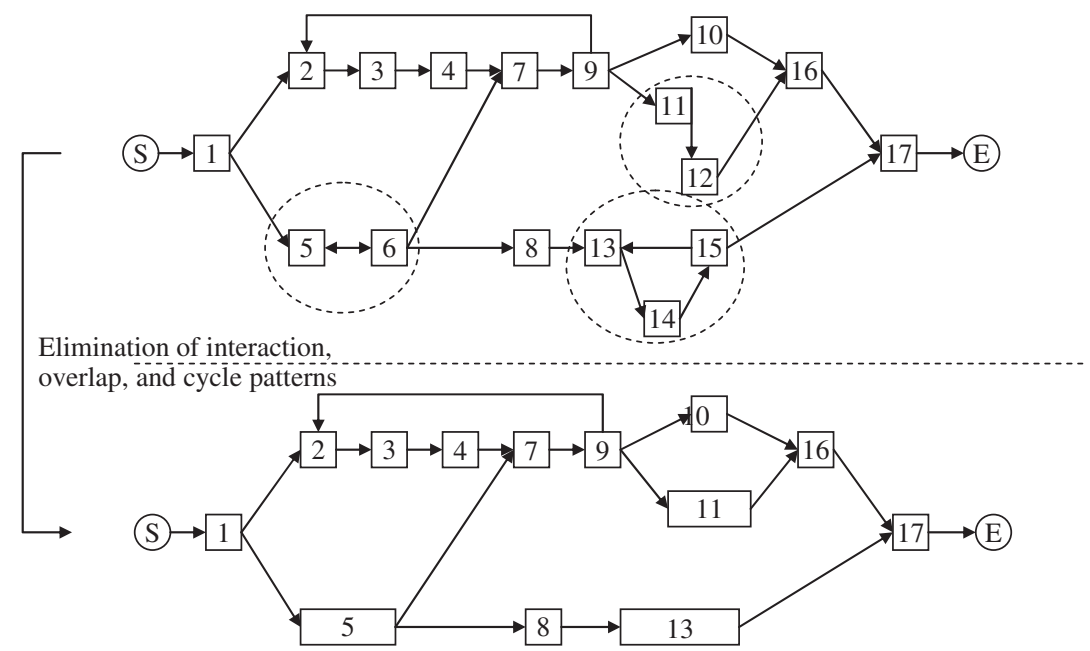

(a) Elimination of the patterns

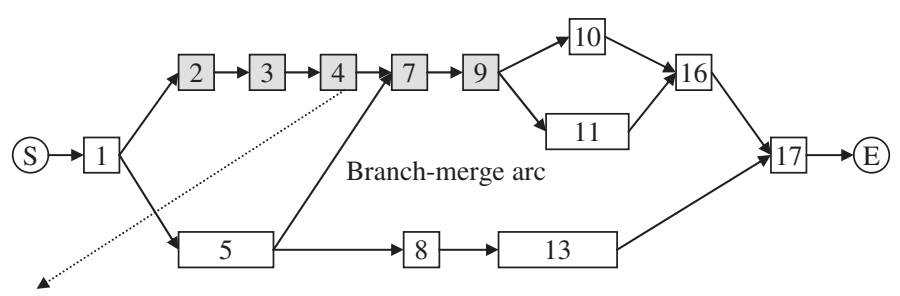

Modified duration $=$ the number of iterations $\times$ duration of the activity

(b) Elimination of the loops

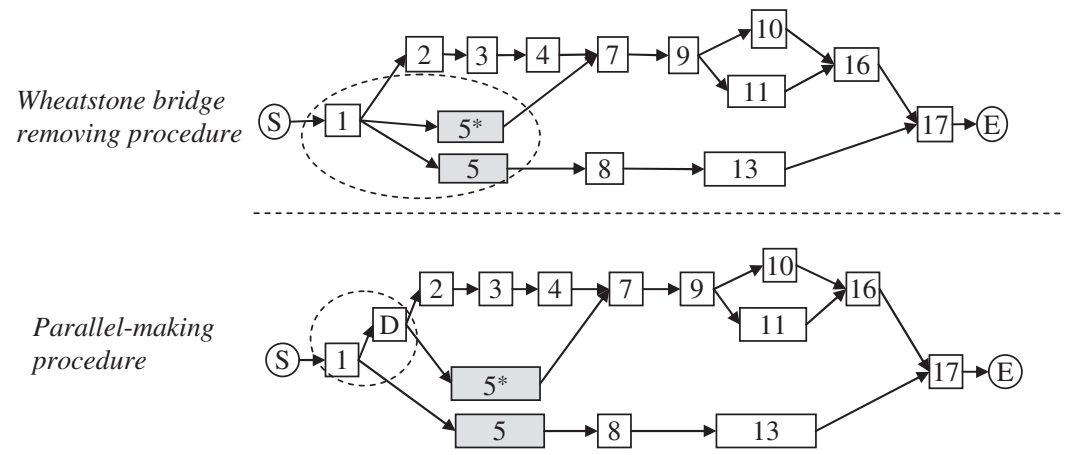

(c) Series-parallel transformation

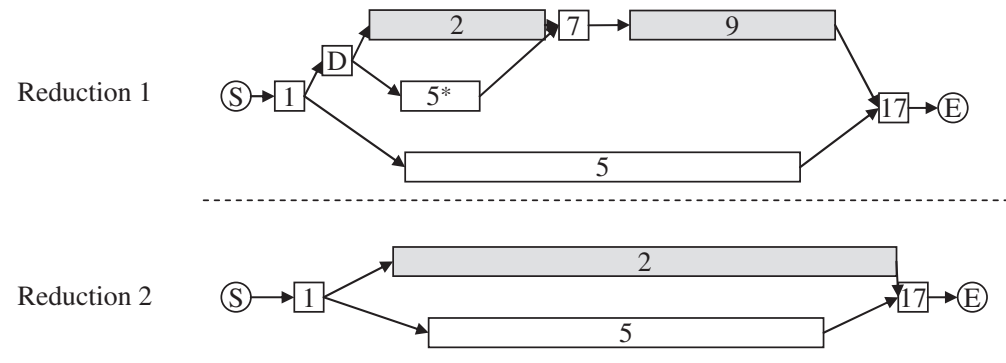

Reduction 3

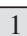

(d) Reduction of the network

Figure 5. PDP-network transformation. 
Table 3. PD process data in Figure 5.

\begin{tabular}{|c|c|c|c|c|c|c|}
\hline No. & Activity name & Predecessor & Successor & Duration & Learning rate & Branch-Merge \\
\hline 1 & Identifying customer needs & - & 2,5 & 3 & 0 & Branch (AND-Syn.) \\
\hline 2 & Review of product development feasibility & 1,9 & 3 & 5 & 0.1 & - \\
\hline 3 & Meeting for checking the feasibility of product development & 2 & 4 & 7 & 0.2 & - \\
\hline 4 & Organizing cross function team (CFT) & 3 & 7 & 4 & 0.1 & - \\
\hline 5 & Review request for product development & 1 & 6 & 20 & 0.3 & - \\
\hline 6 & Receipt of request document of product development & 5 & $5,7,8$ & 11 & 0.4 & Branch (AND-Syn.) \\
\hline 7 & Making a project master plan & 4,6 & 9 & 1 & 0.2 & Merge (AND-Syn.) \\
\hline 8 & Benchmarking & 6 & 13 & 40 & 0.1 & - \\
\hline 9 & Design review & 7 & $2,10,11$ & 4 & 0.3 & Branch (AND-Syn.) \\
\hline 10 & FMEA & 9 & 16 & 31 & 0.2 & - \\
\hline 11 & Release of preliminary specifications & 9 & 12 & 4 & 0.3 & - \\
\hline 12 & Parts developing for a prototype & 11 & 16 & 6 & 0.12 & - \\
\hline 13 & Design specification freezing and releasing & 8 & 14 & 12 & 0.2 & - \\
\hline 14 & Parts developing for mass production & 13 & 15 & 10 & 0.3 & - \\
\hline 15 & Preparing production line & 14 & 13,17 & 5 & 0.1 & - \\
\hline 16 & Production verification test & 10,12 & 17 & 7 & 0.2 & Merge (AND-Syn.) \\
\hline 17 & Pre-production & 15,16 & - & 3 & 0.3 & Merge (AND-Syn.) \\
\hline
\end{tabular}

Step 2. Eliminate the feedback loops with Markov chain analysis. In this step, the probability of the feedback loops is estimated from Activities 9 to 2 with Markov chain analysis. The loop arc of feedback pattern is removed by replacing duration of each activity of feedback pattern with new duration. The new duration can be obtained by the multiplication of the number of iterations and the duration of each activity as shown in Figure 5(b).

Step 3. Transform the PDP-network to series-parallel network with two procedures. The branch-merge arc is eliminated from Activities 5 to 7. For this, dummy activities are used and the series-parallel network is made as shown in Figure 5(c). As a result of the transformation, ENDSM is changed.

Step 4. Estimate the lead time of series-parallel network with analytic formulae of simple branch-merge patterns. Simplifying the series-parallel network is repeated. For this, the activities of each pattern is replaced (no-overlap or branch-merge) with single activity having the same makespan so that there exists only single activity as shown in Figure 5(d). In order to estimate the makespans of branch-merge patterns, the formulae in Jun et al. [6] are used. Finally, the lead time of the whole PD process is found: 104.3 days.

\section{Computational Experiments}

In order to evaluate the performance of SBM-PDP, computational experiments were done with two cases. One is the case that applied the algorithm to a real PD process. The lead time of the PD process of $K$ company that consists of 34 activities including feedback, branch-merge, interaction, and overlap patterns was estimated (Figure 1). The result of the algorithm was 566 days (about 18.6 months). It was known that the real lead time of this PD process was 18 months. This result shows that the proposed approach provides us with a good estimate. The other case used a 180 randomly generated PD process for evaluating this algorithm. For this, five problems were generated for each combination of three levels of the number of activities (35, 40, and 45) and 12 types of branch-merge pattern. As shown in Figure 6, 12 types of branch-merge pattern can be grouped into three parts. One group contains three branch-merge patterns (Types 1-3). Another group contains four (Types 4-7) and the other group has five (Types 8-12). To get appropriate estimates of parameters in each problem such as duration time, transition time, probability of iteration, and so on, related field engineers were interviewed. As a result, the distribution of each parameter for generating a reasonable test problem was made as follows. Here, $\mathrm{DU}(x, y)$ denotes discrete uniform distribution with a range $x$ to $y$.

1. The duration of each activity is generated, with the 0.7 probability from $\mathrm{DU}(1,30)$, with the 0.2 probability from $\mathrm{DU}(31,70)$, and with the 0.1 probability from $\mathrm{DU}(71,90)$.

2. The transition time of each activity is generated from $\mathrm{DU}(1,5)$.

3. The learning rate of each activity is generated from $\mathrm{DU}(0.1,0.6)$.

4. The number of iterations is generated from $\operatorname{DU}(6,11)$.

5. The probability of iteration is generated from $\mathrm{DU}(0.1,0.3)$.

6. The number of interactions is generated from $\mathrm{DU}(1,3)$.

7. The number of cycles is generated from $\operatorname{DU}(0,2)$. 

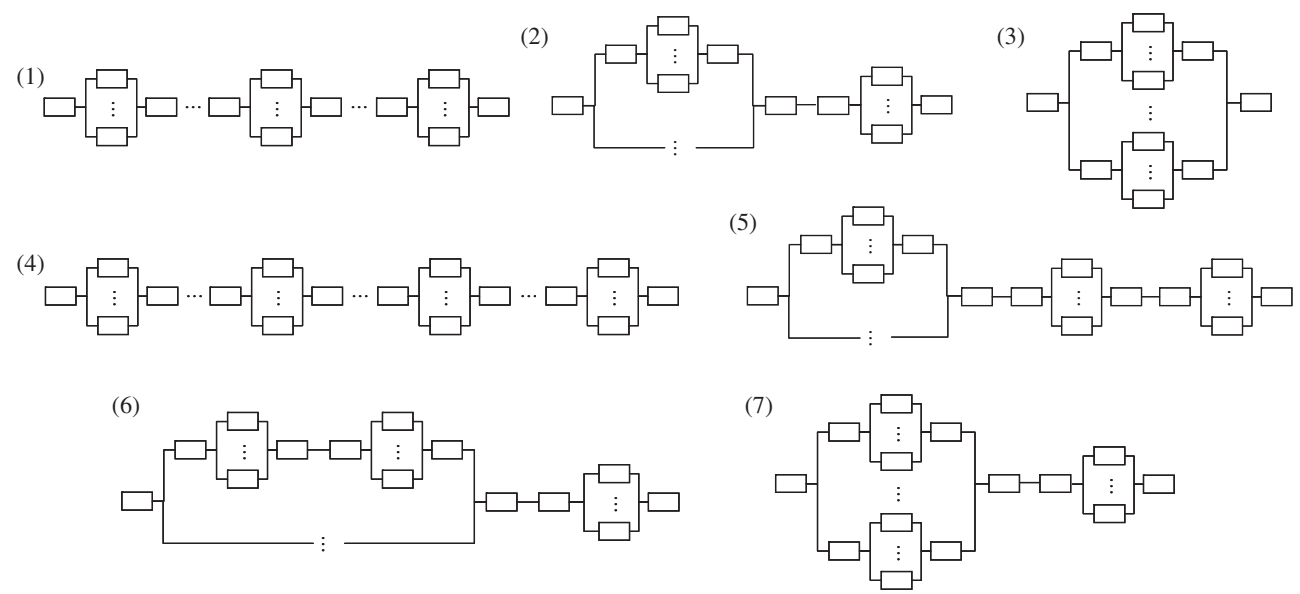

(8)

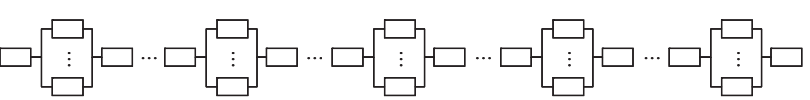

(9)

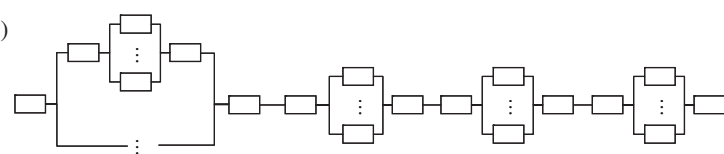

(10)

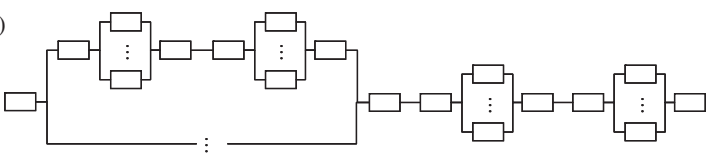

(11)

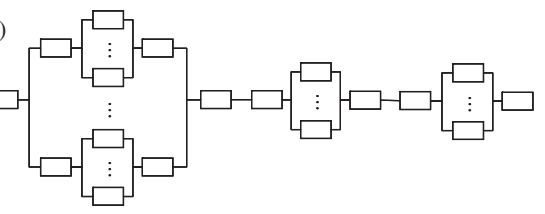

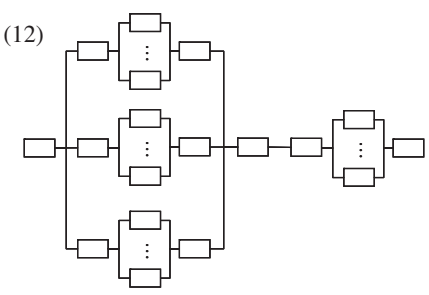

Figure 6. Branch-merge patterns used in the test problems.

8. The number of communications is generated from $\mathrm{DU}(0,1)$.

9. The number of activities in the cycle or communication is generated from $\operatorname{DU}(3,5)$.

10. The number of activities in the feedback is generated from $\mathrm{DU}(2,6)$.

For each test problem, the SBM-PDP was applied to estimate the lead time. The SBM-PDP was compared with simulation results. As the complex PD process considered in this study contains overlap interaction, cycle communication and several types of branch-merge pattern, there are no similar previous estimation methods that can handle this complex PD process. Although there are some methods that deal with some patterns, it is not reasonable to compare them with the method presented because they cannot deal with all patterns that are considered in this study. That is why the comparison of this method with simulations was selected. In the simulation, one can find the best solution in a given same situation, suitable to be compared with the estimates. As a result, the solution of SBM-PDP is compared with the best value among 20 simulation values for each test problem. Parameters or data needed for the simulation model are obtained from the data of each problem. The simulation model is probabilistic because the routing is randomly selected. However, some non-deterministic factors such as exceptional cases and variability of processing times are not considered here.

The algorithm was coded with $C$, and computational experiments were done on a personal computer with a Pentium process operating at $1 \mathrm{GHz}$ clock speed. The test results of 180 randomly generated problems are described in Table 4. Since the average CPU times to implement the heuristic algorithm and each simulation were within $1 \mathrm{~s}$, they are omitted in the test result. For the comparison, a performance measure called the percentage error (PE) is used, which is defined by $100 \cdot\left|E-E_{\text {sim }}\right| / E_{\text {sim }}$, where $E$ and $E_{\text {sim }}$ are the estimated 


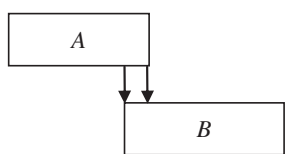

(a) Overlap

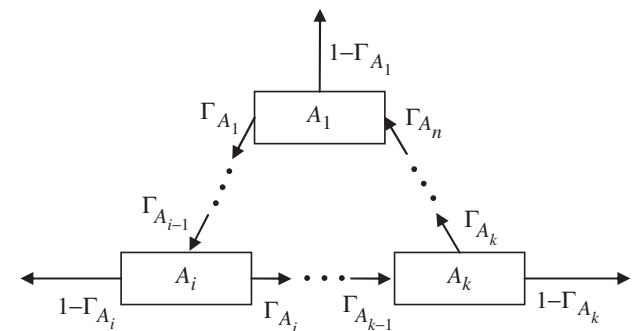

(c) Cycle

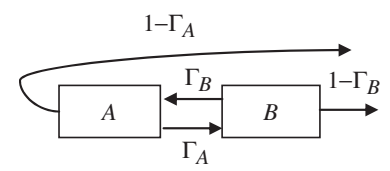

(b) Interaction

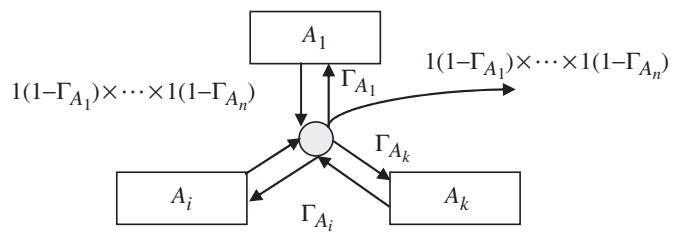

(d) Communication

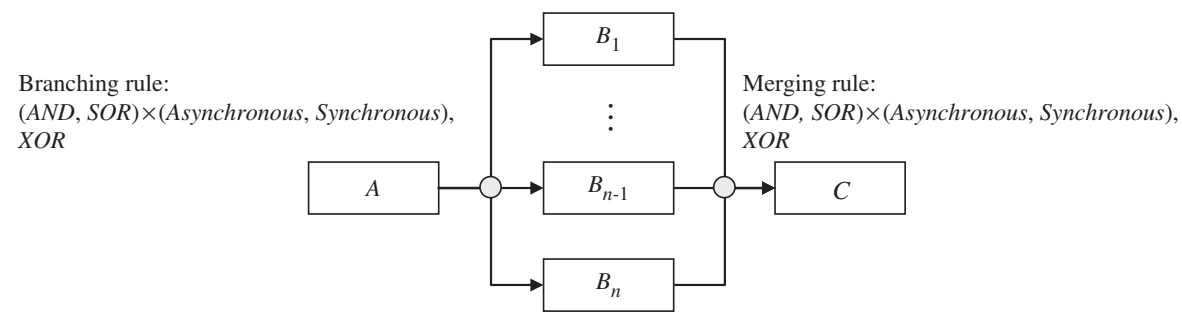

(e) Branching and merging pattern

Figure 7. Patterns.

Table 4. Summary of computational results.

\begin{tabular}{|c|c|c|c|c|c|}
\hline \multirow[b]{2}{*}{ Factor } & & \multicolumn{3}{|c|}{ The number of activities } & \multirow[b]{2}{*}{ Average } \\
\hline & & 35 & 40 & 45 & \\
\hline & 1 & $5.04^{\mathrm{a}}\left(61.53^{\mathrm{b}}\right)$ & $6.70(88.14)$ & 7.83(153.39) & $6.53(101.2)$ \\
\hline & 2 & $7.67(65.17)$ & $6.14(67.84)$ & $7.07(66.35)$ & $6.96(66.45)$ \\
\hline & 3 & $4.35(54.51)$ & 7.54(86.49) & $6.02(61.33)$ & $5.97(67.44)$ \\
\hline & 4 & $6.96(82.40)$ & $6.75(97.69)$ & $7.11(76.10)$ & $6.94(85.40)$ \\
\hline & 5 & 7.90(92.56) & $6.69(53.20)$ & $5.37(100.2)$ & $6.65(82.99)$ \\
\hline & 6 & $6.41(73.49)$ & $6.99(60.83)$ & $7.76(93.11)$ & $7.05(75.81)$ \\
\hline \multirow[t]{6}{*}{ Type of structure } & 7 & $6.45(59.69)$ & $7.39(68.77)$ & $6.13(58.55)$ & $6.66(62.34)$ \\
\hline & 8 & $5.72(81.29)$ & $5.95(90.23)$ & $5.39(77.97)$ & $5.69(83.16)$ \\
\hline & 9 & 7.02(59.77) & $4.61(38.91)$ & $5.13(60.81)$ & $5.59(53.17)$ \\
\hline & 10 & $6.64(57.01)$ & $6.55(72.15)$ & $5.77(76.81)$ & $6.32(68.66)$ \\
\hline & 11 & $6.25(56.80)$ & $7.53(76.23)$ & $7.79(100.4)$ & $7.19(77.81)$ \\
\hline & 12 & $6.70(55.26)$ & 7.24(61.03) & $6.02(44.76)$ & $6.65(53.68)$ \\
\hline Average & & $6.42(65.78)$ & $6.67(75.50)$ & $6.45(78.81)$ & $6.52(73.36)$ \\
\hline
\end{tabular}

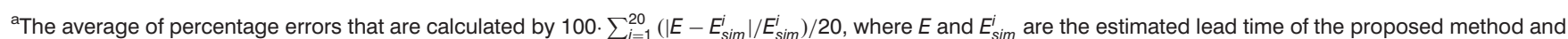
that of the ith simulation, respectively.

${ }^{\mathrm{b}}$ The average standard deviation of simulation values.

lead time of the proposed method and that of the simulation, respectively. This index reflects how well the SBM-PDP estimates the lead time compared with the simulation.
Table 4 shows the average PE and the average standard deviation of simulation values according to the number of activities and the type of structure. The main observation is that the average PE of the 
suggested method is about $6 \%$. The value indicates that there exist some variances of the estimates in simulation. The variance comes from the uncertainty that is due to the fact that the PDP-network has many iterations and selections at the branching or merging. The average standard deviation is also not so small because of the uncertainty of the PD process. However, it is considered that the results are reasonable ones considering the complexity and uncertainty of PD process. Another point found from the test result is that the accuracy of the estimates of SBMPDP is not affected by the number of activities and the types of branch-merge pattern. This tells that the SBM-PDP gives a robust estimate, irrespective of the degree of complexity of the PDP-network. Therefore, this test result provides the evidence that SBM-PDP is useful in estimating the lead time of the whole PD process.

The estimation method presented here can overcome the limitations of previous estimation methods of the lead time of PD process such as PERT and GERT. Although PERT and GERT can provide good estimates in simple cases, they are limited in terms of handling complex PD patterns so that their estimations must be ineffective. On the contrary, the test results show that this method can provide good estimates in a complex PD situations. It is important to estimate the lead time of complex PD process because it is a crucial indicator in PD project management. Project managers can use this method to manage and adjust a PD process plan. A good PD project manager should know how to plan, execute the plan, anticipate problems, and adjust for variances. This method can support project managers in the task of estimating the completion time of a PD project. Although resources, financial factors, and schedule feasibilities in the PD project management should be further considered, this method can be a basis for them.

In spite of several benefits of this method, it is limited in terms of the following: First, this study does not cover all PD processes and behaviors. Since this method is based on the structured PD network that consists of combinations of patterns, there might be difficulty in interpreting some PD structures with mixed types of patterns. Moreover, there might be a lack of completeness of the ontology of PD process patterns. This study might not include all specific PD patterns that can arise in a complex PD environment. For example, the development of information communication technologies leads to changes in the working patterns of PD engineers. Hence, there might be new patterns (relations) that this method cannot incorporate. Finally, in the analysis of a PD process, considering only the time factor excluding resource constraints and cost might be a limitation of this study, although it is a tricky problem.

\section{Conclusions}

In this study, a heuristic algorithm has been presented to estimate the lead time of the PD process. To evaluate the proposed algorithm, a case study and computational experiments have been done. From these results, one can see that the algorithm can be successfully employed in estimating the lead time of a complex PD process. This model and several assumptions may not describe all situations occurring in the PD process because of the intractability and the general characteristics of the model. Despite the restrictions, this model allows one to analyze a complex PD process with time aspect, unlike PERT. Moreover, its model is more tractable than GERT. It can play an important role in planning and managing of the PD project by helping the engineering managers analyze and evaluate the alternative project structure.

This research can be extended in several directions. For example, a more improved and efficient method may be devised and used in estimating the lead time of a complex PD process. One may also build a quantitative model that estimates not only developing time but also developing cost. Moreover, it may be a valuable thing to develop an estimation model with resource constraints. In addition, a computer-based analysis tool may be needed to support the efficient decision making.

\section{Appendix}

\section{A.1 Notation}

To maintain brevity and consistency, the following notations are used.

$i \quad$ Activity index, $i \in\left\{A_{1}, A_{2}, \ldots, A_{n} ; B_{1}, B_{2}, \ldots, B_{n} ; C\right\}$

$D_{i}$ The duration of an activity $i$

$T_{i, j}$ The elapsed time between the completion of an activity $i$ and the start of an activity $j$

$N$ The expected number of iterations

$N_{i}$ The expected number of iterations of an activity $i$

$\Gamma_{i}$ The probability that an activity $i$ is repeated, $0 \leq \Gamma_{i}<1$

$l_{y}(x)$ The learning effect function of an activity $y$ in the $x$ th iteration

$\omega$ The makespan of a pattern

$\Delta t$ Overlapping time

$G_{1} \quad$ Expected time spent on reworks 


\section{A.2 Overlap}

The estimated makespan for an overlap pattern is

$$
\omega=D_{A}+D_{B}-\Delta t+G_{1} .
$$

For more details of $\Delta t$ and $G_{1}$, refer to Jun et al. [6].

\section{A.3 Interaction}

The estimate for the makespan of an interaction pattern (Figure 7(b)) is

$$
\begin{aligned}
w= & \sum_{x=1}^{\left\lfloor N_{A}\right\rfloor}\left(D_{A} \cdot l_{A}(x)+T_{B, A}\right)-T_{B, A} \\
& +\sum_{x=1}^{\left\lfloor N_{B}\right\rfloor}\left(D_{B} \cdot l_{B}(x)+T_{A, B}\right) \\
& +\left(N_{A}-\left\lfloor N_{A}\right\rfloor\right)\left\{D_{A} \cdot l_{A}\left(\left\lfloor N_{A}^{*}\right\rfloor\right)+T_{B, A}\right\} \\
& +\left(N_{B}-\left\lfloor N_{B}\right\rfloor\right)\left\{D_{B} \cdot l_{B}\left(\left\lfloor N_{B}^{*}\right\rfloor\right)+T_{A, B}\right\} .
\end{aligned}
$$

where

$$
\begin{aligned}
N_{A} & =1 \cdot\left(1-\Gamma_{B}\right)+\sum_{n=2}^{\infty} n\left(\Gamma_{A} \Gamma_{B}\right)^{n-2} \cdot \Gamma_{B}\left(1-\Gamma_{A} \Gamma_{B}\right) \\
& =1-\Gamma_{B}+\Gamma_{B} \cdot\left(2-\Gamma_{A} \Gamma_{B}\right) /\left(1-\Gamma_{A} \Gamma_{B}\right), \\
0 & <\Gamma_{A}, \Gamma_{B}<1 .
\end{aligned}
$$

$$
\begin{aligned}
N_{B} & =\sum_{n=1}^{\infty} n\left(\Gamma_{A} \Gamma_{B}\right)^{n-1} \cdot\left(1-\Gamma_{A} \Gamma_{B}\right) \\
& =1 /\left(1-\Gamma_{A} \Gamma_{B}\right), 0<\Gamma_{A}, \Gamma_{B}<1 .
\end{aligned}
$$

In (2), $\lfloor y\rfloor$ is the largest integer that does not exceed $y$, and $N_{A}^{*}=N_{A}+0.5$ and $N_{B}^{*}=N_{B}+0.5$ are integers rounded-off of $N_{A}$ and $N_{B}$, respectively. The estimated makespan is the sum of the total time spent in activities $A$ and $B$ with $\left\lfloor N_{A}\right\rfloor$ and $\left\lfloor N_{B}\right\rfloor$ iterations (first three terms) and corrective terms to compensate for the round-off error of iterations.

\section{A.4 Cycle}

The expected number of iterations for an activity $i$ is

$$
\begin{aligned}
& \text { For } k=1,2, \ldots, n, \\
& \qquad \begin{aligned}
N_{A_{k}}= & 1+\sum_{i=1}^{\infty} i \cdot\left(\Gamma_{1} \ldots \Gamma_{n}\right)^{i-1} \cdot \sum_{j=1}^{n} \Pi_{m=0}^{k-2} \Gamma_{\sigma^{m}(j)} \cdot \Pi_{p=1}^{j-1} \Gamma_{p} \\
& \times\left(1-\Gamma_{\sigma^{k-1}(j)}\right) \\
= & \frac{1}{\left(1-\Gamma_{1} \ldots \Gamma_{n}\right)^{2}} \sum_{j=1}^{n} \Pi_{m=0}^{k-2} \Gamma_{\sigma^{m}(j)} \cdot \Pi_{p=1}^{j-1} \Gamma_{p} \cdot\left(1-\Gamma_{\sigma^{k-1}(j)}\right)
\end{aligned}
\end{aligned}
$$

where $\Pi_{p=1}^{0} \Gamma_{p}=1$ and $\Pi_{m=0}^{-1} \Gamma_{\sigma_{(j)}^{m}}=1$ for any $j$. Here $\Gamma_{k}$ indicates $\Gamma_{A_{k}}$, and $\sigma$ is a bijective function: $\{1,2, \ldots, n\} \rightarrow\{1,2, \ldots, n\}$ such that $\sigma(i)=i+1, i=1$,

Table 5. Analytical models of simple branching and merging patterns [6].

Type

AND(Syn.)-AND(Syn.)

AND(Syn.)-AND(Asyn.)

AND(Asyn.)-AND(Syn.)

AND(Asyn.)-AND(Asyn.)

AND(Syn.)-SOR(Syn.)

AND(Syn.)-SOR(Asyn.)

AND(Asyn.)-SOR(Syn.)

AND(Asyn.)-SOR(Asyn.)

SOR(Syn.)-SOR(Syn.)

SOR(Syn.)-SOR(Asyn.) SOR(Asyn.)-SOR(Syn.) SOR(Asyn.)-SOR(Asyn.) SOR(Syn.)-AND(Syn.) SOR(Syn.)-AND(Asyn.) SOR(Asyn.)-AND(Syn.) SOR(Asyn.)-AND(Asyn.) AND(Syn.)-XOR

AND(Asyn.)-XOR SOR(Syn.)-XOR

SOR(Asyn.)-XOR XOR-XOR

\section{Expressions}

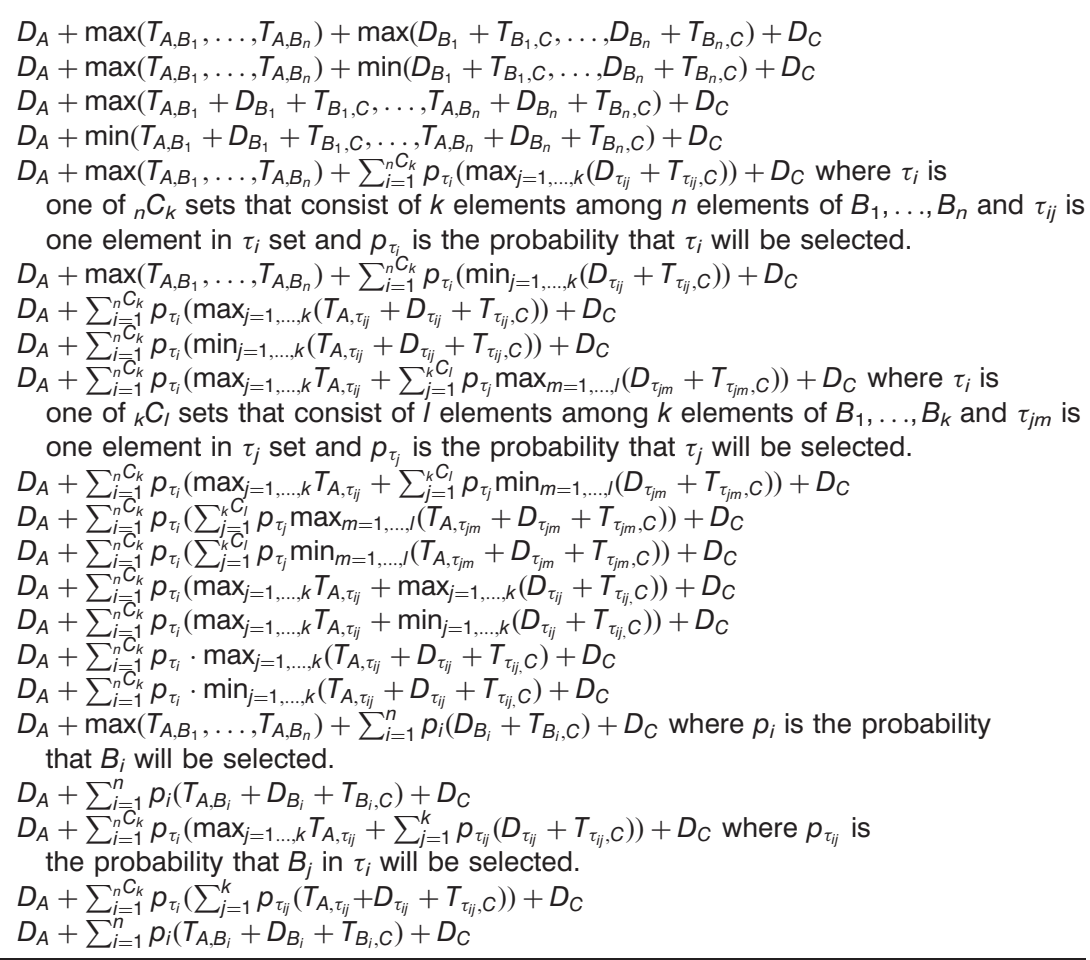


$2, \ldots, n-1$ and $\sigma(n)=1$. In addition, $\sigma^{k}(i)$ is a $k$ th recursive relation such that $\sigma^{k}(i)=i+k$. Accounting for the savings from learning when the same activities are repeated, one can estimate the makespan as:

$$
\begin{aligned}
w= & \sum_{k=1}^{n} \sum_{x=1}^{\left\lfloor N_{A_{k}}\right\rfloor}\left(D_{A_{k}} \cdot l_{A_{k}}(x)+T_{A_{k-1}, A_{k}}\right)-T_{A_{n}, A_{1}} \\
& +\sum_{k=1}^{n}\left(N_{A_{k}}-\left\lfloor N_{A_{k}}\right\rfloor\right)\left(D_{A_{k}} \cdot l_{A_{k}}\left(\left\lfloor N_{A_{k}}^{*}\right\rfloor\right)+T_{A_{k-1}, A_{k}}\right)
\end{aligned}
$$

where $N_{A_{k}}^{*}=N_{A_{k}}+0.5$ and $T_{A_{0}, A_{1}}=T_{A_{n}, A_{1}}$. The second term is used to compensate for the overestimated elapsed time. Refer to Figure 7(c).

\section{A.5 Communication}

The expected number of iterations in a communication pattern (Figure $7(\mathrm{~d})$ ) is

$$
\begin{aligned}
N & =\sum_{k=1}^{\infty} k\left(1-\Pi_{i=1}^{n}\left(1-\Gamma_{A_{i}}\right)\right)^{k-1} \cdot \Pi_{i=1}^{n}\left(1-\Gamma_{A_{i}}\right) \\
& =1 / \Pi_{i=1}^{n}\left(1-\Gamma_{A_{i}}\right) .
\end{aligned}
$$

With probability $\left(1-\Pi_{i=1}^{n}\left(1-\Gamma_{A_{i}}\right)\right)$, the pattern fails to disseminate the final output in a given iteration and must repeat the activities. Then, the estimated makespan of a communication pattern is computed by adding up the duration of each activity until the pattern terminates while taking into account learning effect:

$$
\begin{aligned}
w= & \sum_{x=1}^{\lfloor N\rfloor} \max _{i=1, \ldots, n} D_{A_{i}} \cdot l_{A_{i}}(x) \\
& +(N-\lfloor N\rfloor)) \cdot \max _{i=1, \ldots, n} D_{A_{i}} \cdot l_{A_{i}}\left(\left\lfloor N^{*}\right\rfloor\right)+N \cdot \sigma
\end{aligned}
$$

where $N^{*}=N+0.5$ and $\sigma$ is the average communication time per iteration.

\section{A.6 Branching and Merging}

The makespan of a lot of types of branch and merge (Figure 7(e)) can be estimated with formulae as described in Table 5.

\section{References}

1. Johnson, E.W., Brockman, J.B. and Vigeland, R. (1996a). Sensitivity Analysis of Iterative Design Processes, In: IEEE/ ACM International Conference on Computer-Aided Design, ICCAD-96, Digest of Technical Papers, pp. 142-145.

2. Johnson, E.W., Castillo, L.A. and Brockman, J.B. (1996b). Application of a Markov Model to the Measurement, Simulation, and Diagnosis of an Iterative Design Process,
In: Proceedings of the 33rd IEEE Design Automation Conference, pp. 185-188.

3. Belhe, U. and Kusiak, A. (1996a). Modeling Relationships Among Design Activities, Journal of Mechanical Design, 118: 454-460.

4. Dodin, B. (1985). Bounding the Project Completion Time Distribution in PERT Net Works, Operations Research, 33(4): 862-881.

5. Grunder, O., Baptiste, P. and Barakat, O. (1995). An Approach to Model and Evaluate Innovation and Concurrent Engineering Projects, In: Proceedings of the INRIA/IEEE Symposium on Emerging Technologies and Factory Automation, 1: 155-163.

6. Jun, H.B., Ahn, H.S. and Suh, H.W. (2005). On Identifying and Estimating the Cycle Time of Product Development Process, IEEE Transactions on Engineering Management, 52(3): 336-349.

7. Adlakha, V.G. and Kulkarni, V.G. (1989). A Classified Bibliography of Research on Stochastic PERT Networks: 1966-1987*, INFOR, 27(3): 272-296.

8. Lawrence, F.P. and Cochran, J.K. (1995). A New Computational Approach for Project Management Networks, Computers and Industrial Engineering, 29(1-4): 339-343.

9. Yassine, A.A., Falkenburg, D. and Chelst, K. (1999b). Engineering Design Management: An Information Structure Approach, International Journal of Production Research, 37(13): 2957-2975.

10. Taylor III, B.W. and Moore, L.J. (1980). R\&D Project Planning with Q-GERT Network Modeling and Simulation, Management Science, 26(1): 44-59.

11. Krishnan, V., Eppinger, S. and Whitney, D.E. (1995) Accelerating Product Development by the Exchange of Preliminary Product Design Information, Journal of Mechanical Design, 117: 491-498.

12. Kusiak, A., Wang, J., He, D.W. and Feng, C. (1995). A Structured Approach for Analysis of Design Processes, IEEE Transactions on Components, Packing, and Manufacturing Technology-Part A, 18(3): 664-673.

13. Smith, R.P. and Eppinger, S.D. (1997b). Identifying Controlling Features of Engineering Design Iteration, Management Science, 43(3): 276-293.

14. Steward, D.V. (1981). The Design Structure System: A Method for Managing the Design of Complex Systems, IEEE Transactions on Engineering Management, 28(3): 71-74.

15. Smith, R.P. and Eppinger, S.D. (1997a). A Predictive Model of Sequential Iteration in Engineering Design, Management Science, 43(8): 1104-1120.

16. Pritsker, A. and Happ, W. (1966). GERT: Graphical Evaluation and Review Technique, Part I, Fundamental, Journal of Industrial Engineering, 17(5): 267-274.

17. Belhe, U. and Kusiak, A. (1995). Resource Constrained Scheduling of Hierarchically Structured Design Activity Networks, IEEE Transactions on Engineering Management, 42(2): 150-158.

18. Belhe, U. and Kusiak, A. (1993). Performance Analysis of Design Process Using Timed Petri Nets, Concurrent Engineering: Research and Applications, 1(3): 147-152.

19. Kara, S., Kayis, B. and Kaebernick, H. (2001). Concurrent Resource Allocation (CRA): A Heuristic for Multi-project Scheduling with Resource Constraints in Concurrent Engineering, Concurrent Engineering: Research and Applications, 9(1): 64-73. 
20. Yan, H.-S., Wang, Z. and Jiang, M. (2002). A Quantitative Approach to the Process Modeling and Planning in Concurrent Engineering, Concurrent Engineering: Research and Applications, 10(2): 97-111.

21. Eppinger, S.D., Nukala, M.V. and Whitney, D.E. (1997). Generalised Models of Design Iteration Using Signal Flow Graphs, Research in Engineering Design, 9: 112-123.

22. Belhe, U. and Kusiak, A. (1996b). Scheduling Design Activities with a Pull System Approach, IEEE Transactions on Robotics and Automation, 12(1): 15-21.

23. Smith, R.P. and Eppinger, S.D. (1998). Deciding Between Sequential and Concurrent Tasks in Engineering Design, Concurrent Engineering: Research and Applications, 6(1): 15-25.

24. Kusiak, A. and Park, K. (1990). Concurrent Engineering: Decomposition and Scheduling of Design Activities, International Journal of Production Research, 28(10): 1883-1900.

25. Kusiak, A. and Wang, J. (1993). Efficient Organizing of Design Activities, International Journal of Production Research, 31(4): 753-769.

26. Ha, A.Y. and Porteus, E.L. (1995). Optimal Timing of Reviews in Concurrent Design for Manufacturability, Management Science, 41(9): 1431-1447.

27. Loch, C.H. and Terwiesch, C. (1998). Communication and Uncertainty in Concurrent Engineering, Management Science, 44(8): 1032-1048.

28. Yassine, A.A., Chelst, K.R. and Falkenburg, D.R. (1999a). A Decision Analytic Framework for Evaluating Concurrent Engineering, IEEE Transactions on Engineering Management, 46(2): 144-157.

29. Krishnan, V., Eppinger, S.D. and Whitney, D.E. (1997). A Model-based Framework to Overlap Product Development Activities, Management Science, 43(4): 437-451.

30. Schweickert, R., Giorgini, M. and Dzhafarov, E. (2000). Selective Influence and Response Time Cumulative Distribution Functions in Serial-parallel Task Networks, Journal of Mathematical Psychology, 44(4): 504-535.

31. Kao, E.P.C. (1997). An Introduction to Stochastic Processes, Duxbery Press.

\section{Hon-Bae Jun}

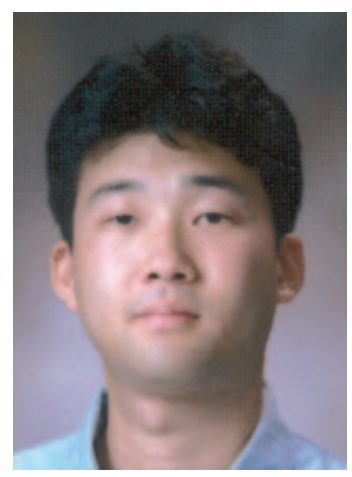

Hon-Bae Jun received his $\mathrm{PhD}$ in Industrial Engineering (IE) from Korea Advanced Institute of Science and Technology (KAIST) at Daejeon, Korea (2003), as well as MS in IE (1997) from the same institute. He graduated in Applied Statistics from Yonsei University of Seoul, Korea (1995). He is now working, from 2004, as an assistant researcher of laboratory for Computer-Aided Design and Production at Swiss Federal Institute of Technology in Lausanne (EPFL). At present he takes part in the PROMISE (PROduct lifecycle Management and Information tracking using Smart Embedded systems) project (EU and IMS project). His current research interests are Product Lifecycle Management (PLM), Lifecycle information modeling, Product Recovery/EOL management, and Auto-ID/RFID technologies.

\section{Jin-Young Park}

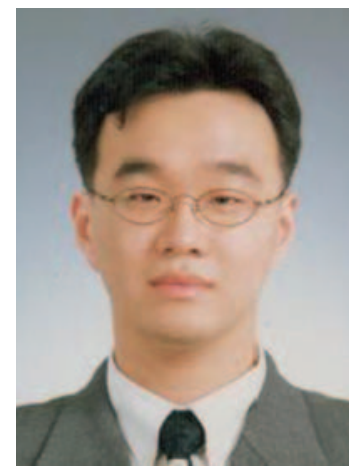

Jin-Young Park received his BS degree in Mathematics from Sung Kyun Kwan University (SKKU) in 1998 and MS degree in Mathematics from Korea Advanced Institute of Science and Technology (KAIST) in 2003. He has been studying, since 2003, as a $\mathrm{PhD}$ candidate of Concurrent Engineering (CE) at the laboratory in Industrial Engineering (IE) at KAIST and he has also been working, since 2005, as a researcher of Production Engineering Research Institute in LG Electronics (LGPERI). His current research interests are Business Process Reengineering (BPR), Ontology-based knowledge engineering, Concurrent Engineering (CE), Artificial Intelligence (AI), Virtual Reality (VR), Design for Cost (DFC), and Product Lifecycle Management (PLM).

\section{Hyo-Won Suh}

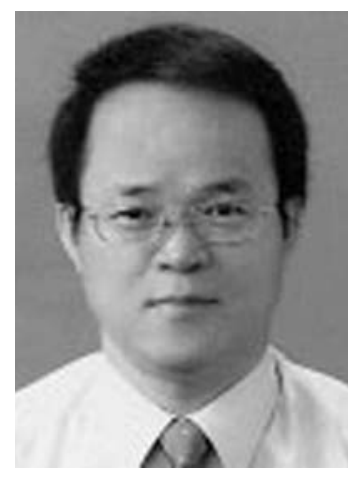

Hyo-Won Suh received his BS degree in Mechanical Engineering from Yonsei University, MS degree in Mechanical Engineering from Korea Advanced Institute of Science and Technology (KAIST), and $\mathrm{PhD}$ in Industrial Engineering from West Virginia University. He is an associate professor at the Department of Industrial Engineering, KAIST. His research interests include collaborative engineering, workflow and business process management, product data management, and ontology-based knowledge engineering. 